\title{
Resistance distribution in the hopping percolation model
}

\author{
Yakov M. Strelniker, Shlomo Havlin, Richard Berkovits, and Aviad Frydman \\ Minerva Center, Jack and Pearl Resnick Institute of Advanced Technology, \\ and Department of Physics, Bar-Ilan University, 52900 Ramat-Gan, Israel
}

(Dated: October 30, 2018)

\begin{abstract}
We study the distribution function, $P(\rho)$, of the effective resistance, $\rho$, in two and threedimensional random resistor network of linear size $L$ in the hopping percolation model. In this model each bond has a conductivity taken from an exponential form $\sigma \propto \exp (-\kappa r)$, where $\kappa$ is a measure of disorder, and $r$ is a random number, $0 \leq r \leq 1$. We find that in both the usual strong disorder regime $L / \kappa^{\nu}>1$ (not sensitive to removal of any single bond) and the extreme disorder regime $L / \kappa^{\nu}<1$ (very sensitive to such a removal) the distribution depends only on $L / \kappa^{\nu}$ and can be well approximated by a log-normal function with dispersion $b \kappa^{\nu} / L$, where $b$ is a coefficient which depends on the type of the lattice.
\end{abstract}




\section{INTRODUCTION}

The concepts and methods of percolation theory are widely used to explain many phenomena in physics, classical as well as quantum (for review see e.g. Refs. 1, 2, 3]). The canonical model for studying transport properties of disordered systems is percolation on a lattice. Usually it is also assumed that the conductivity between neighboring lattice sites may be defined as either finite, or zero (i.e., either conducting or insulating) without loss of generality. This model [which we denote as the bond (or site) percolation] has been extensively studied and is understood quite well. For the description of the nearest neighbor hopping in granular materials it is much more natural to define the conductivity between two neighboring lattice sites (labeled as "i" and "j") by $\sigma_{i j} \propto \exp \left[-r_{i j} / r_{0}-\epsilon_{i j} / k_{B} T\right]$, where $r_{i j}$ is the distance between the two sites, $r_{0}$ is the scale over which the wave-function outside the grain decays, $\epsilon_{i j}$ is the energy difference between grains, and $T$ is the temperature. Here we neglect the thermal hopping term (high temperature regime) and consider only nearest neighbor hopping. This behavior may be captured by a lattice model for which [4, 5, 6, 6, 8]

$$
\sigma_{i j}=\sigma_{0} \exp [-\kappa r(i j)]
$$

where $\kappa$ is a measure of disorder, $r(i j)$ is a random number taken from uniform distribution in the range $(0,1)$, and $\sigma_{0}$ is a dimension coefficient [4, 5]. We shall name this model the hopping percolation model.

One might expect that such small differences in the formulation of the disorder in the $\sigma_{i j}$ (namely, $\sigma_{i j}=0,1$ or $\sigma_{i j}=\sigma_{0} \exp [-\kappa r(i j)]$ ) will lead to no important difference in the global conductance properties of these systems. Quite surprisingly, recent experiments on the conductance of granular material [9] might suggest otherwise. Specifically, the number of red bonds (which are critical for current) expected in framework of the traditional percolation theory is proportional to $L^{1 / \nu}$, where $\nu$ is the percolation correlation critical exponent and $L$ is the system size [10]. Thus, for a typical experimental set-up of $10^{9}$ grains, one expects $10^{2}$ red bonds. On the other hand, measurements of transport through Ni granular ferromagnets, indicates a much lower number of red bonds (typically of order one) 9]. In a recent paper 5] we have attributed this difference to the fact that the estimation of the number of red bonds $\left(L^{1 / \nu}\right)$ is based on the bond percolation model, while for the hopping percolation we expect a transition to a regime of extreme strong disorder in which a single red bond governs 


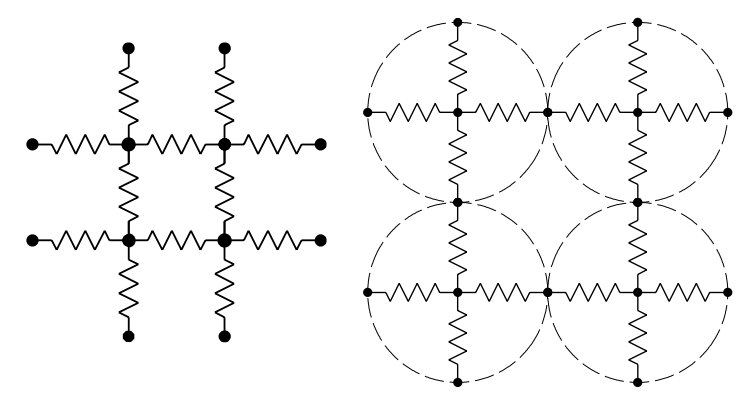

a.

b.

FIG. 1: (a) A square bond percolation net of resistors with random resistivity given by Eq. (1), where $(i j)$ denotes the bond between sites $i$ and $j$. (b) A site percolating network. The resistivity of all four resistors within a dashed circle is determined by a single random number $r(i j)$, where $i j$ denotes the labeling of the grid point $i, j$.

the behavior of the system. The onset of this regime scales as to $\kappa / L^{1 / \nu}$.

It is important to note that in contrast to the traditional bond (or site) percolation model, in which the system is either a metal or an insulator, for the hopping percolation model the system always conducts some current. Hopping conductivity (i.e., exponential local resistance) (11) is always associated with strong disorder. As was shown in Ref. [5], there are two regimes within this strong disorder: a regime which is not sensitive to the removal of a single bond, as expected from the usual percolation theory, termed the usual strong disorder regime [1]. While for even stronger disorder a regime which is very sensitive to the removal of a specific single bond exists, denote as extreme disorder. In the extreme disorder regime a single bond can determine the transport properties of the entire macroscopic system [5, 9$]$.

The remainder of this paper is arranged as follows. In Section „ we describe our model and the numerical approach. In Section „ we present some numerical results, followed by a brief discussion in Section.

\section{MODEL}

We perform large-scale Monte Carlo simulations for calculating transport in these systems. We build a bond-percolating Miller-Abrahams like resistor network (see Fig. 1 and 


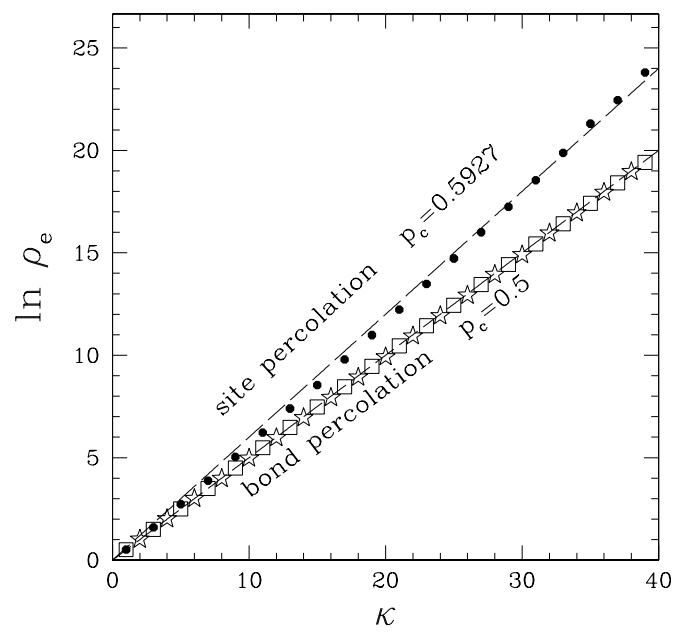

FIG. 2: A semi-log plot of the averaged resistance $\rho_{e}$ vs. $\kappa$. In the case of site percolation $\left(p_{c}=0.592746\right)$ the slope of the curve is close to 0.6 , while for the case of bond percolation $\left(p_{c}=0.5\right)$ this slope is equal to 0.5 [cf. with Eq. (2)]. The system sizes shown are: $L=20$ (solid circles), 40 (open squeres), 100 (open stars).

Ref. 5, 12, 13, 14), but assume the conductivity of each resistor to have the form given in Eq. (11). Then solve the corresponding set of linear Kirchhoff equations and calculate the total effective resistance $\rho_{e}$ for two dimensional (2D) and three dimensional (3D) networks [5, 12, 13, 14].

We begin by calculating the average effective conductivity $\sigma_{e}$. The approximate expression 1, 2, 4] for the effective conductivity $\sigma_{e}$, of a random resistor network [13] with local conductivities given by Eq. (11), in $2 \mathrm{D}$ is

$$
\sigma_{e}=\sigma_{0} e^{-p_{c} \kappa}
$$

In Fig. 2 we show this dependence (in terms of resistivity $\rho_{e}=1 / \sigma_{e}$ ) for both site and bond percolations (see Fig. [1) for different lattice sizes. In Ref. [6] it was shown that in the limit $\kappa \rightarrow \infty$, Eq. (2) is exact. It is easy to show that in the case of $2 \mathrm{D}$ random resistor bond network (for which $p_{c}=0.5$ ), Eq. (2) follows immediately from the Keller-Dykhne theorem and is exact for arbitrary $\kappa$ [15]. Similarly this results can be found in framework of the symmetric self-consistency effective-medium approximation (EMA) rewritten for many component composite [16]. From Eq. (2) follows that the effective conductivity $\sigma_{e}$ depends on $\kappa$ and does not depend on the system size $L$. For finite $L$, Eq. (2) represents the mean 
conductivity over all configurations of the disordered system.

Next we study the fluctuations of the resistance, $\rho=1 / \sigma$, from the mean value $\rho_{e}=1 / \sigma_{e}$ for individual systems of finite size $L$. We perform numerical calculations of the probability distribution function $P(\rho)$ (i.e., the probability that the total resistance of the system is $\rho$ ) as well as the variance $\operatorname{var}(\rho)$ as a function of $L$ and $\kappa$. As shown in Ref. [5], the relative variance (in contrast to $\rho_{e}$ ), strongly depends on $L$ and $\kappa$ only through the scaled variable $h \equiv L / \kappa^{\nu}$. Here $\nu$ is the critical exponent of the percolation correlation length $\xi \propto\left(p-p_{c}\right)^{-\nu}$ (in $2 \mathrm{D} \nu=4 / 3 \simeq 1.33$, while in $3 \mathrm{D} \nu \simeq 0.88$ [1, 2, 3] ). It was also shown [5] that $h$ describes the transition from strong disorder $(h>1)$ to extreme disorder $(h<1)$.

\section{RESULTS}

Here we present numerical results suggesting that $P(\rho)$ also depends only on $L / \kappa^{\nu}$. In order to verify and to quantify this hypothesis, we study numerically $P(\rho)$ for systems of different sizes $L$ and different disorder $\kappa$, but with the same value of $h$ (see also Ref. 17]). In Figs. 3 and 4 we show $P(\rho)$ vs. $\rho$ for the cases of strong and extreme disorder. All data corresponding to the same parameter $h$ scale according to the same law [see Figs. 3)(c) and [3(g)]. Thus, our results suggest that $P(\rho)$ is a function of both $\rho / \rho_{e}$ and $h$, i.e.,

$$
P(\rho)=\frac{1}{\rho_{e}} f\left(\frac{\rho}{\rho_{e}} ; h\right) .
$$

Here $h$ determines the form of the function and $P(\rho)$ for a fixed $h$ depends only on $\rho / \rho_{e}$. Fig. 3(c) suggests that in the strong disorder, $h$ controls the width or standard deviation of the rescaled distribution. Since the standard deviation increases when $h$ decreases, we assume that the standard deviation $\delta \simeq b \rho_{e} / h=b \rho_{e} \kappa^{\nu} / L$, where $b$ is a parameter which depends on the type of lattice. Indeed, when we plot in Fig. 通(d) $P\left(\rho / \rho_{e}\right) \delta$ vs. $\left(\rho-\rho_{e}\right) / \delta$, a collapse of the two plots shown in Fig. 3(c) is obtained. The functional form obtained in Fig. 3(d) suggests that the probability distribution can be approximated by a Gaussian

$$
P(\rho) \simeq(\sqrt{2 \pi} \delta)^{-1} \exp \left[-\left(\rho-\rho_{e}\right)^{2} / 2 \delta^{2}\right]
$$

Indeed, the dashed line in Fig. 3(d) represents a good fit to the Gaussian given by Eq. (41). However, Eq. (4) can not approximate the asymmetric form of $P(\rho)$ at extreme disorder [see Figs. 3(g) and 3(h)]. We suggest, as will be justified below, that $P(\rho)$ can be approximated 

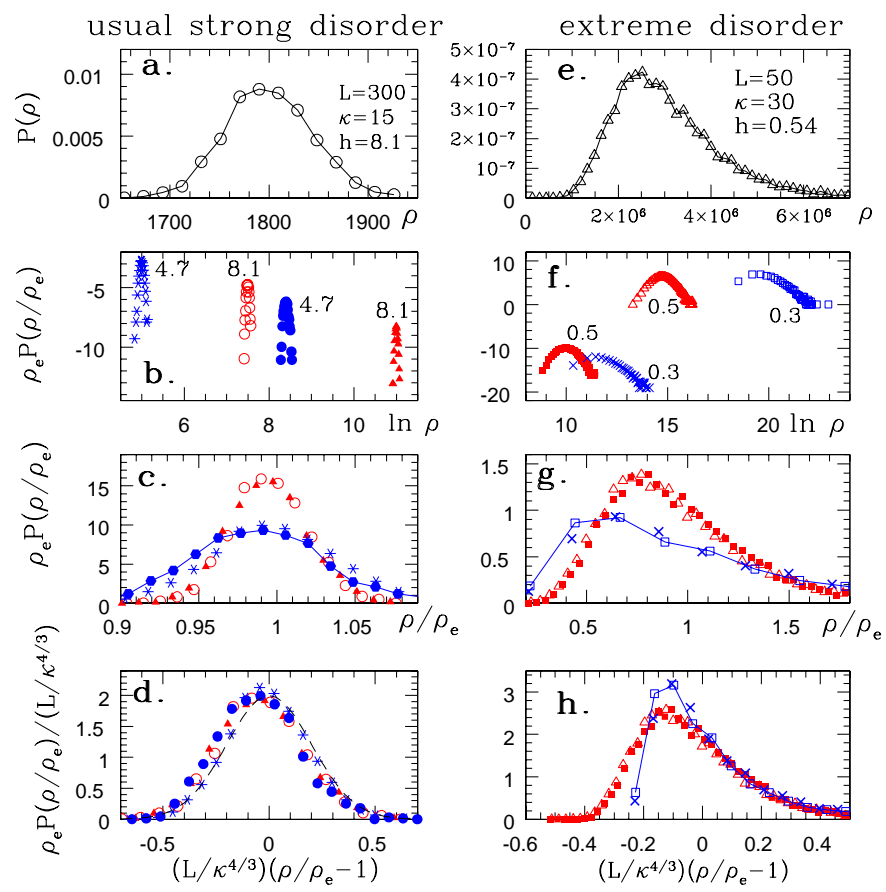

FIG. 3: (a)-(d) The probability distribution for the case of usual strong disorder $(h>1)$. (a) A typical form of $P(\rho)$ vs. $\rho$ for $h=8.1$. (b) Similar to (a), but shown in log-log scale for $L=300$, $\kappa=15, h=8.1$ (०), $L=500, \kappa=22, h=8.1(\mathbf{\Lambda}), L=100, \kappa=10, h=4.7$ (*), and $L=200$, $\kappa=16.84, h=4.7(\bullet)$. (c) Scaling plots $\rho_{e} P\left(\rho / \rho_{e}\right)$ vs. $\rho / \rho_{e}$ for the results shown in (b). Only the data with the same value of $h$ scale to a unique function. (d) Scaling plots of $\rho_{e} P\left(\rho / \rho_{e}\right) /\left(L / \kappa^{\nu}\right)$ vs. $\left(L / \kappa^{\nu}\right)\left(\rho / \rho_{e}-1\right)$ lead to the collapse of the data shown in (b) and (c). The dashed line in (d) is the Gaussian distribution (4) with $b=0.2$. (e)-(h) Similar to (a)-(d), but for systems with extreme disorder $(h<1): L=50, \kappa=30, h=0.54(\triangle), L=30, \kappa=20.4, h=0.54(\mathbf{\square}), L=40$, $\kappa=40, h=0.3(\square)$ and $L=20, \kappa=23.78, h=0.3(\times)$. (h) Similar process like (d), does not lead to collapse in the case of extreme disorder. The lines connecting the points for $h=4.7$ in (c) and for $h=0.3$ in (g) and (h) are guides to the eye.

(in all regimes of disorder) by the log-normal form

$$
P(\rho) \simeq \frac{1}{\sqrt{2 \pi} \mu \rho} \exp \left[-\frac{\ln ^{2}\left(\rho / \rho_{e}\right)}{2 \mu^{2}}\right],
$$

where $\mu=\delta / \rho_{e}=b \kappa^{\nu} / L$. In fact, Eq. (15) includes also the usual strong disorder case, since in the latter case $\ln ^{2}\left(\rho / \rho_{e}\right) \simeq\left(\rho / \rho_{e}-1\right)^{2}$ and Eq. (5I) reduces to the Gaussian form (41)), while at extreme disorder $(\mu \gg 1)$ the exponent function in Eq. (5) tends to 1, and $P(\rho)$ transforms to the power-like dependence $\sim 1 / \rho$ (see Fig. 4). 

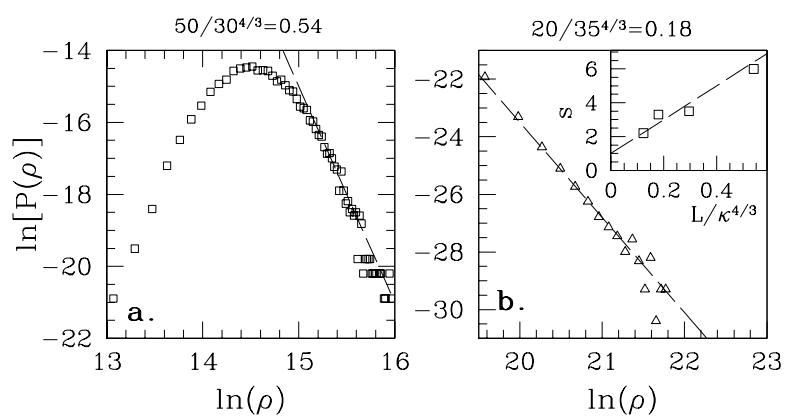

FIG. 4: A log-log plot of $P(\rho)$ vs. $\rho$. By increasing disorder the log-normal distribution transforms to the power-law $\rho^{-s}$. (a) $L=50, \kappa=30(h=0.54)$. (b) $L=20, \kappa=35(h=0.18)$. Inset: The exponent $s$ (of the power law $\rho^{-s}$ ) vs. $h$. By increasing disorder (i.e., decreasing $h$ ) the exponent $s$ tends to $1 . L=50, \kappa=30(h=0.54) ; L=14, \kappa=18.19(h=0.3) ; L=20, \kappa=35(h=0.18)$; $L=14, \kappa=35(h=0.12) . \nu=4 / 3$.

From Eq. (5) it follows that at $\mu \rightarrow 0$, the distribution function $P(\rho)$ reduces to a deltafunction: $\lim _{\mu \rightarrow 0} P(\rho)=\frac{1}{\rho} \lim _{\mu \rightarrow 0} \frac{1}{\sqrt{2 \pi} \mu} e^{-\frac{\ln \left(\rho / \rho_{e}\right)^{2}}{2 \mu^{2}}}=\frac{1}{\rho} \delta\left(\ln \rho-\ln \rho_{e}\right)=\delta\left(\rho-\rho_{e}\right)$. Therefore, at $\mu \rightarrow 0$ (i.e., $\kappa \rightarrow 0$ or $L \rightarrow \infty$ ) the total resistivity of the system is exactly $\rho_{0} e^{p_{c} \kappa}$ and has no size dependence: $\lim _{\mu \rightarrow 0} \int \rho P(\rho) d \rho=\rho_{e}$.

It should be noted that a log-normal distribution of resistances is found in quantum models of hopping conductivities (see e.g., Ref. [18] and references therein), while here it is demonstrated for classical exponential disorder (II). Moreover, our result (51) yield the specific analytical form of $P(\rho)$, which includes the dependence on $\kappa$ and $L$ for all regimes of disorder.

In Fig. 5 (a) we test Eq. (15) by comparing it to simulation results. It is shown that the numerical results of the $2 \mathrm{D}$ resistance $\rho P\left(\rho / \rho_{e}\right) /\left(L / \kappa^{\nu}\right)$ scale vs. $\left(\rho / \rho_{e}\right)^{L / \kappa^{\nu}}$, as predicted by Eq. (5) for both strong and extreme disorder. A similar plot is presented in Fig. [5) (b) for a 3D lattice. Although for the 3D case Eq. (2) is not exact (since Keller-Dykhne theorem exists only in 2D), nevertheless the approximated expression $\sigma_{e} \simeq \sigma_{0} \kappa^{\nu} e^{-p_{c} \kappa}$ is known 4, 6], resulting in the distribution law (5). Since in Eq. (2) the parameter $\kappa$ appears with the prefactor $p_{c}$, we should expect that $p_{c}$ enters into the parameter $\mu$ of Eq. (15) as $\mu=$ $\alpha\left(p_{c} \kappa\right)^{\nu} / L$. Comparing the values $b=0.2$ observed for the square bond percolation lattice $\left(p_{c}=0.5\right)$ and $b=0.18$ for the cubic site percolation $\left(p_{c}=0.3116\right)$, we find that $\alpha=0.503$. The dependence of $\mu$ on $p_{c}$ is in agreement with result of Ref. 19]. These results strongly 

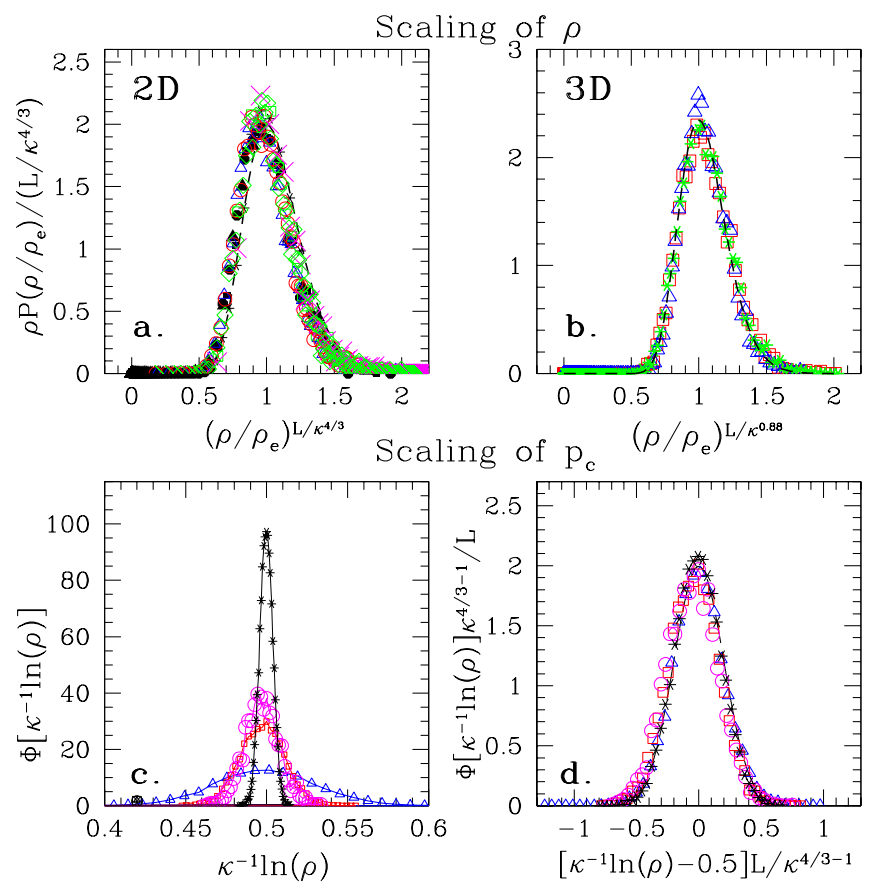

FIG. 5: (a) A scaling plot of $\rho P\left(\rho / \rho_{e}\right)$ vs. $\left(\rho / \rho_{e}\right)^{L / \kappa^{\nu}}$ of the data plotted in Fig. 3] (2D case). The dashed line represents the analytical result (5), with $b=0.2$ and $\nu=4 / 3$ for 9 systems with $L=500, \kappa=22(\boldsymbol{\Delta}) ; L=300, \kappa=15(\boldsymbol{\square}) ; L=200, \kappa=10(\bullet) ; L=100, \kappa=10(*) ; L=50$, $\kappa=30(\triangle) ; L=40, \kappa=40(\square) ; L=30, \kappa=20($ ( ); $L=20, \kappa=40(\times) ; L=20, \kappa=30(\diamond)$; (b) Similar to (a), but for 3D, with $b=0.18$ and $\nu=0.88$ for three systems with $L=20, \kappa=15$ $(\square) ; L=26, \kappa=15(\triangle) ; L=10, \kappa=6.8(*)$. $(h=1.85)$. The dashed line is the analytical result, Eq. (5). (c) $\Phi\left(p_{c}\right)=\Phi[\ln (\rho) / \kappa]$ vs. $p_{c}$, where $p_{c}=\kappa^{-1} \ln (\rho)$ for different values of the ratios $L / \kappa^{\nu}$ : $L=100, \kappa=10, h=4.68(*) ; L=60, \kappa=30, h=0.65$ (०); $L=40, \kappa=20, h=0.74(\square)$; $L=20, \kappa=30, h=0.22(\triangle) ;(\mathrm{d})$ Scaling $\Phi[\ln (\rho) / \kappa] /\left(L / \kappa^{\nu-1}\right)$ vs. $[\ln (\rho) / \kappa-0.5]\left(L / \kappa^{\nu-1}\right)$ with $\nu=4 / 3$. The values of the ratios $L / \kappa^{\nu}$ are the same as in (c). The dashed line is the analytical fit, as derived from Eq. (5).

support our proposition that Eq. (5) describes well the distribution in all ranges of disorder.

The variance $\operatorname{var}(\rho)$ can be expressed as $\left\langle\rho^{2}\right\rangle-\rho_{e}^{2}$, where $\left\langle\rho^{n}\right\rangle=\int_{\rho_{\min }}^{\rho_{\max }} \rho^{n} P(\rho) d \rho$, and $\rho_{e}=\langle\rho\rangle$. For large enough $\kappa,\left\langle\rho^{n}\right\rangle=\int_{0}^{\infty} \rho^{n} P(\rho) d \rho=\rho_{0} e^{n \ln \rho_{e}+\frac{1}{2} n^{2} \mu^{2}}$ and the relative variance takes the form

$$
[\operatorname{var}(\rho)]^{1 / 2} / \rho_{e}=\left[e^{\mu^{2}}\left(e^{\mu^{2}}-1\right)\right]^{1 / 2}
$$

(see Ref. 20]). Fig. 6 presents numerical results showing that the relative variance scales as 

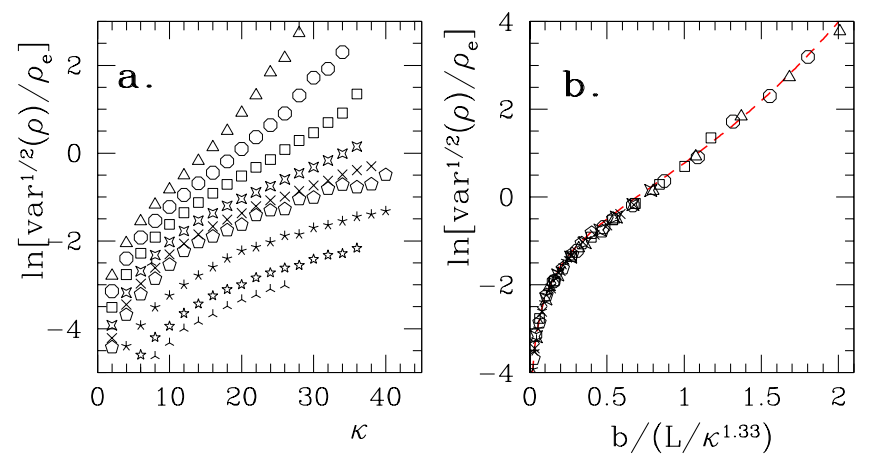

FIG. 6: (a) A semi-log plot of the relative variance $[\operatorname{var}(\rho)]^{1 / 2} / \rho_{e}$ vs. $\kappa$ for various sizes of the system: $L=10,14,20,30,40,50,100,200,300$ (from top to bottom). (b) A semi-log scaling plot of the same quantity vs. $\mu=b \kappa^{\nu} / L$, where $b=0.2, \nu=4 / 3$. The dashed line represents Eq. (6).

a function of $\mu=b \kappa^{\nu} / L$ in accordance with Eq. (6).

Next we shall present analytical arguments for the log-normal distribution (515). According to the central limit theorem [20], if the values of $\ln \rho$ are normally distributed, then the values of $\rho$ should follow the log-normal distribution. Assuming $\ln \rho=\kappa p_{c}$ [see Eq. (2)] for all $\rho$, the distribution $P\left(\kappa^{-1} \ln \rho\right)$ is simply the distribution of the percolation threshold $\Phi\left(p_{c}\right)$ which is normally distributed (e.g., Refs. 21]). Indeed, in Fig. 5(c) we show that $\Phi\left[\kappa^{-1} \ln (\rho)\right]$ approximately follows a normal distribution centered at $p_{c}=0.5$. Thus, the distribution of $\rho$ should be log-normal as in Eq. (5).

Using the above assumption $\ln \rho=\mu / \kappa=\kappa p_{c}$, it is possible to evaluate the distribution $\Phi\left(p_{c}\right)$ and its standard deviation $\delta_{p_{c}}$. One can write a simple relation $\Phi\left(p_{c}\right) d p_{c}=\Phi^{\prime}(y) d y$, where $y=\kappa p_{c}$, and get $\Phi\left(p_{c}\right)=\Phi^{\prime}(y) \frac{d y}{d p_{c}}=\kappa \Phi(y)$. Therefore, $\Phi\left(p_{c}\right)=\kappa \Phi^{\prime}(\ln \rho) \sim$ $\kappa \mu^{-1} \exp \left[-\kappa^{2}\left(p_{c}-\bar{p}_{c}\right)^{2} / 2 \mu^{2}\right]=\delta_{p_{c}}^{-1} \exp \left[-\left(p_{c}-\bar{p}_{c}\right)^{2} / 2 \delta_{p_{c}}^{2}\right]$, where $\bar{p}_{c}$ is the mean value of the percolation threshold and $\delta_{p_{c}}=\mu / \kappa$ is the standard deviation of $\Phi\left(p_{c}\right)$. This form of $\Phi\left(p_{c}\right)$ is supported by our numerical simulations shown in Figs. 5 (c) and 5 (d).

This specific form for $\delta_{p_{c}}=b \kappa^{\nu-1} / L$ in the hopping percolation model should be compared to $\delta_{p_{c}}=L^{-1 / \nu}$ known for the bond percolation model [10, 21]. This further emphasizes the differences between the bond percolation model considered in Ref. 21] and the hopping percolation model considered here. 


\section{SUMMARY}

In summary, we find the specific form of the resistance distribution in the hopping percolation model. For all ranges of strong disorder $\kappa$ and lattice sizes $L$, the distribution is $\log$-normal and depends only on the ratio $\kappa^{\nu} / L$, where $\nu$ is the correlation exponent for the bond percolation case. Assuming the relation $\rho=\exp \left(\kappa p_{c}\right)$ for finite systems leads to a variance of $p_{c}, \delta_{p_{c}}=\kappa^{\nu-1} / L$, which is different from $\delta_{p_{c}}=L^{-1 / \nu}$ known for the bond percolation model [21]. Our results may be relevant to ac conductivity measurements in such systems. By appropriate choice of frequency one can detect regions of size smaller than $\kappa^{\nu}$, where a crossover in behavior from extreme to usual strong disorder behavior is expected.

This research was supported in part by grants from the US-Israel Binational Science Foundation, the Israel Science Foundation, and the KAMEA Fellowship program of the Ministry of Absorption of the State of Israel.

[1] B. I. Shklovskii and A. L. Efros, Electronic Properties of Doped Semiconductors, Springer (1984).

[2] D. Stauffer and A. Aharony, Introduction to Percolation Theory, Taylor and Francis, London (1992).

[3] A. Bunde and S. Havlin, (Eds.), Fractals and Disordered Systems, Springer-Verlag, Berlin (1996).

[4] V. Ambegaokar, B. I. Halperin, and J. S. Langer, Phys. Rev. B 4, 2612 (1971).

[5] Y. M. Strelniker, R. Berkovits, A. Frydman, and S. Havlin, Phys. Rev. E 69, 065105(R) (2004).

[6] S. Tyc and B. I. Halperin, Phys. Rev. B 39, R877 (1989).

[7] P. Le Doussal, Phys. Rev. 39, R881 (1989).

[8] A.E. Morozovskii and A. A. Snarskii, Pis'ma Zh. Eksp. Teor. Fiz. 56, 272 (1992) [JETP Lett. 56, 268 (1992)]; A.K. Sarychev and F. Brouers, Phys. Rev. Lett. 73, 2895 (1994); T.H. Skaggs, Physica B 338, 266 (2003).

[9] A. Cohen, A. Frydman, and R. Berkovits, Solid State Comm. 129, 291 (2004).

[10] A. Coniglio, J. Phys. A: Math. Gen. 15, 3829 (1982). 
[11] In Ref. 5] we named these regimes "strong disorder" and "weak disorder" as used commonly in the optimization literature [see e.g., M. Cieplak, A. Maritan, and J. R. Banavar, Phys. Rev. Lett. 72, 2320 (1994), M. Porto, N. Schwartz, S. Havlin, and A. Bunde, Phys. Rev. E 60, R2448 (1999), and N. Schwartz, M. Porto, S. Havlin, and A. Bunde, Physica A 266, 317 (1999)]. Here we use a different terminology in order to be consistent with the common terminology used for hopping transport.

[12] A. Miller and E. Abrahams, Phys. Rev. 120, 745 (1960).

[13] S. Kirkpatrick, Rev. Mod. Phys. 45, 574 (1973).

[14] A. K. Sarychev, D. J. Bergman, and Y. M. Strelniker, Phys. Rev. B 48, 3145 (1993).

[15] Following Keller and Dykhne [see J. B. Keller, J. Math. Phys. 5, 548 (1964); A. M. Dykhne, Zh. Eksp. Teor. Fiz. 59, 110 (1970) [Sov. Phys.-JETP 32, 63 (1971)]; Y. M. Strelniker and D. J. Bergman, Phys. Rev. B 61, 6288 (2000) and Y. M. Strelniker and D. J. Bergman, Phys. Rev. B 67, 184416 (2003)], $\sigma_{e}$ of $n$-component composite (with local conductivities $\sigma_{i}$ ) and a similar quantity of the dual system (with $\sigma_{i}^{\prime}=\sigma_{i}^{-1}$ ) are connecting as: $\sigma_{e}\left(\sigma_{1}, \sigma_{2}, \ldots \sigma_{n}\right) \sigma_{e}\left(\sigma_{1}^{-1}, \sigma_{2}^{-1}, \ldots \sigma_{n}^{-1}\right)=1$. Rewriting Eq. (10) as $\sigma_{i j} \sim e^{-0.5 \kappa} e^{-\kappa[r(i j)-0.5]}=$ $e^{-0.5 \kappa} e^{-\kappa \tilde{r}}[$ where $\tilde{r} \in(-0.5,0.5)]$ and taking into account that $\sigma_{e}$ is a homogeneous function [see e.g., D. J. Bergman and D. Stroud, Solid State Physics 45, 147 (1992)], i.e., that any factor can be taken out from the brackets, we get: $e^{-0.5 \kappa} \sigma_{e}\left(\left\{e^{-\kappa \tilde{r}}\right\}\right) e^{0.5 \kappa} \sigma_{e}\left(\left\{e^{\kappa \tilde{r}}\right\}\right)=1$. Since sequences $\left\{e^{-\kappa \tilde{r}}\right\}$ and $\left\{e^{\kappa \tilde{r}}\right\}$ are self-dual, $\sigma_{e}\left(\left\{e^{-\kappa \tilde{r}}\right\}\right)=1$. Multiplying this by $e^{-0.5 \kappa}$, we get Eq. (2). [See also L.G. Fel and K.M. Khanin, J. Stat. Phys. 108, 1015 (2002)]. The numerical verification of Eq. (2) is shown in Fig. 2]

[16] Following R. Juretschke, R. Landauer, and J. A. Swanson, J. Appl. Phys. 27, 838 (1956), D. A. G. Bruggeman, Ann. Physik (Leipzig) 24, 636 (1935), and D. J. Bergman and Y. M. Strelniker, Phys. Rev. B 60, 13016 (1999), we write $\int_{\sigma_{\min }}^{\sigma_{\max }} P(\sigma)\left[\left(\sigma-\sigma_{e}\right) /\left(\sigma+a \sigma_{e}\right)\right] d \sigma=0$, where $a=z / 2-1$ and $z=4$ is the number of bonds at each node [13]. According to Eq. (11), $\sigma_{\min }=\sigma_{0} e^{-\kappa}, \sigma_{\max }=\sigma_{0}$, and $P(\sigma)=1 / \kappa \sigma$. Substituting this into the EMA and taking the integral, we get Eq. (2).

[17] Z. Wu, E. Lopez, S.V. Buldyrev, L.A. Braunstein, S. Havlin, and H. E. Stanley, Phys. Rev. E 71, $045101(2005)$.

[18] A. Cohen, Y. Roth, and B. Shapiro, Phys. Rev. B 38, 12125 (1988); E. Abrahams, P. W. Anderson, D. C. Licciardello, and T. V. Ramakrishnan, Phys. Rev. Lett. 42, 673 (1979). 
[19] T. Kalisky, private communication.

[20] J. Aitchison, and J.A.C. Brown, The Lognormal Distribution, Cambridge, At the University Press, 1969.

[21] M. E. Levinshtein, B. I. Shklovskii, M. S. Shur, and A. L. Efros, Zh. Eksp. Teor. Fiz. 69, 386 (1975) [Sov. Phys. JETP 42, 197 (1975)]; P. J. Reynolds, H. E. Stanley, and W. Klein, Phys. Rev. B 21, 1223 (1980); S. Kirkpatrick, AIP Conf. Proc. 40, 99 (1978). 